\title{
Permanent magnets for the return loop of the Cornell-Brookhaven energy recovery linac test accelerator
}

\author{
S. Brooks@, ' G. Mahler, J. Cintorino, J. Tuozzolo®, and R. Michnoff \\ Brookhaven National Laboratory, Upton, Long Island, New York 11973, USA
}

(Received 18 August 2020; accepted 16 November 2020; published 30 November 2020)

\begin{abstract}
214 neodymium permanent magnets have been manufactured for the fixed-field, multiturn return loop of the Cornell-Brookhaven Energy recovery linac Test Accelerator at Cornell University. There are 5 types of quadrupole and combined-function gradient magnets using a variant of the circular Halbach design. These are made out of $\mathrm{NdFeB}$ material and glued into an aluminium housing with water channels for temperature stabilization. The $\mathrm{NdFeB}$ wedges and magnet construction were done by outside companies, while the final "tuning" using inserts containing 64 iron rods per magnet was done at BNL over a period of about 6 months. Average relative field errors of $2.2 \times 10^{-4}$ were achieved on the beam region. The magnet strengths vary by type but are of order $10 \mathrm{~T} / \mathrm{m}$ for quadrupole component and up to $0.3 \mathrm{~T}$ for the dipole. This paper reports on the field quality and timeline achieved in this production process.
\end{abstract}

DOI: 10.1103/PhysRevAccelBeams.23.112401

\section{MAGNET TYPES AND QUANTITIES}

The different magnet types required for the CornellBrookhaven Energy recovery linac Test Accelerator (CBETA) [1,2] fixed-field accelerator (FFA) return loop are specified in Table I. This loop transports $42,78,114$, and $150 \mathrm{MeV}$ electron beams simultaneously using an alternating gradient lattice with $44.4 \mathrm{~cm}$ cell length and overall curvature radius as strong as $5.1 \mathrm{~m}$ ([2] section 2.6). Simultaneous transport of this wide momentum ratio is enabled by high gradients in all magnets, producing low dispersion and enabling different energies to find areas of differing field with minimal offset from the pipe centre $(\leq 25 \mathrm{~mm})$.

The straight section is a simple FODO lattice containing alternating QF, QD magnets, while the curved parts contain QF alternating with a BD-type magnet. These horizontally defocusing magnets also contain a dipole field, which removes the need for an extra bending element in the cell and further optimizes the dispersion function. This gives a lattice known as the nonscaling FFA, introduced by Trbojevic [3]. Phase advances in the cell fall with energy from 0.368 for the $42 \mathrm{MeV}$ horizontal plane to 0.042 for the $150 \mathrm{MeV}$ vertical.

The BDH and QFH magnets are half-length versions of $\mathrm{BD}$ and $\mathrm{QF}$, used once each at the ends of the accelerator for better matching. That leaves five distinct magnet cross sections as shown in Fig. 1.

\footnotetext{
*sbrooks@bnl.gov
}

Published by the American Physical Society under the terms of the Creative Commons Attribution 4.0 International license. Further distribution of this work must maintain attribution to the author(s) and the published article's title, journal citation, and DOI.
Q-type magnets are quadrupoles, while BD-type are combined function bending and gradient. The sequence $\mathrm{BD}, \mathrm{BDT} 2, \mathrm{BDT} 1, \mathrm{QD}$ gradually decreases the bending component to zero to allow the adiabatic transition from curved arc to straight as shown in Fig. 2. The transitional magnets have larger aperture so that further intermediate values of the dipole, on a continuous range, may be obtained by displacing them horizontally.

Figure 3 shows part of the return loop installed in the hall. While the overall curvature is visible, the permanent magnets are mainly hidden inside the iron window-frame electromagnetic correctors that compensate for placement errors when the magnets are surveyed.

The initial guideline for integrated field accuracy was $10^{-3}$ relative error in the $R \leq 25 \mathrm{~mm}$ good field region, which eventually became formalized as the magnet acceptance criteria in Table II. The Brookhaven rotating coil measures the integrated field to $10^{-5}$ reproducibility, explaining why the goals of Table I have up to six significant figures.

\section{MAGNET SHAPE}

The magnets are based on a 16-segment Halbach [4] design (Fig. 4). For the combined-function magnets such as BD (Fig. 5), the wedge thicknesses and magnetization angles were optimized to give the combined field directly. This uses less permanent magnet (PM) material than nesting a Halbach dipole and quadrupole.

\section{A. Combined function design}

The origin of the lopsided shape of the magnet in Fig. 5 can be explained in terms of superposition. The left half of Fig. 6 shows a Halbach quadrupole nested inside a dipole, 
TABLE I. Magnet specifications.

\begin{tabular}{lrccccc}
\hline \hline Magnet type & Count & Dipole $(\mathrm{T})$ & Gradient $(\mathrm{T} / \mathrm{m})$ & Length $(\mathrm{mm})$ & Aperture radius $(\mathrm{mm})$ & Good field radius $(\mathrm{mm})$ \\
\hline QF & 107 & 0 & -11.5624 & 133 & 43.1 & 25 \\
BD & 32 & -0.3081 & 11.1475 & 122 & 40.1 & 25 \\
BDT2 & 20 & -0.2543 & 11.1475 & 122 & 44.938 & 25 \\
BDT1 & 28 & -0.1002 & 11.1475 & 122 & 49.085 & 25 \\
QD & 27 & 0 & 11.1434 & 122 & 40.1 & 25 \\
QFH & 1 & 0 & -11.5624 & 66.5 & 43.1 & 25 \\
BDH & 1 & -0.3081 & 11.1475 & 61 & 40.1 & 25 \\
\hline \hline
\end{tabular}

which is a valid way of producing a combined-function field since PM material has $\mu_{r} \simeq 1$, making the inner ring transparent to the field from outside. However, looking at the magnetization directions on the right-hand side of this magnet, it becomes clear it is inefficient because pieces at the same position in the two rings are producing opposing fields that cancel.

The right half of Fig. 6 shows what happens if the two rings are combined so that each wedge contains the vector sum of the magnetizations of the dipole and quadrupole parts. This would also work as combined-function magnet but is difficult to realize in practice because PM materials typically have a fixed value of $B_{r}$, or a small range, so that the magnitude of the magnetization vector cannot be continuously varied to zero. If instead, the radial thickness of each wedge is varied in proportion to the magnitude of these vectors, while the magnetization angle is retained, a design similar to Fig. 5 is produced.

This sort of shape is familiar from existing work on combined-function superconducting magnets. Sarma [5] provides many examples of the shapes produced by combining different multipole winding arrangements, including dipole + quadrupole (Fig. 3 in that paper) that resembles the magnet shape used here. J-PARC built combined-function superconducting magnets [6] with asymmetric coil arrangements, although the relationship

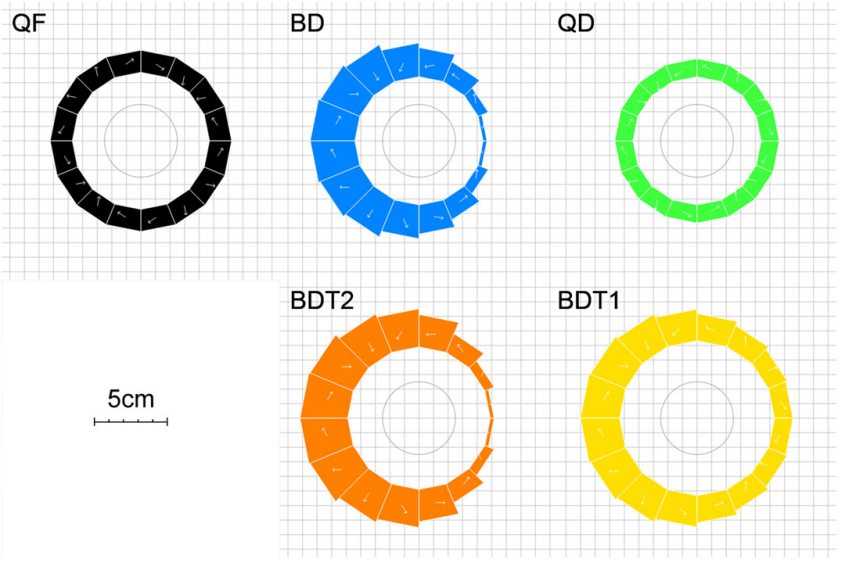

FIG. 1. Permanent magnet cross sections with $1 \mathrm{~cm}$ grid. to the ideal shape is less clear since their conductors are grouped into equal-thickness blocks for ease of assembly. The idea of using asymmetric coil arrangements to generate combined function fields goes back even further [7]. It should not be surprising that superconducting magnets share optimal geometries with permanent magnets because permanent magnets can be modeled as sheet currents flowing around their edges, making both types of "pure current" magnet.

The relationship between the vector magnitudes on the right of Fig. 6 and the wedge thicknesses in Fig. 5 is not exactly linear and becomes less so as the wedges become thicker and the effect of material distance from the center of the magnet becomes significant. Thus, the exact angles and

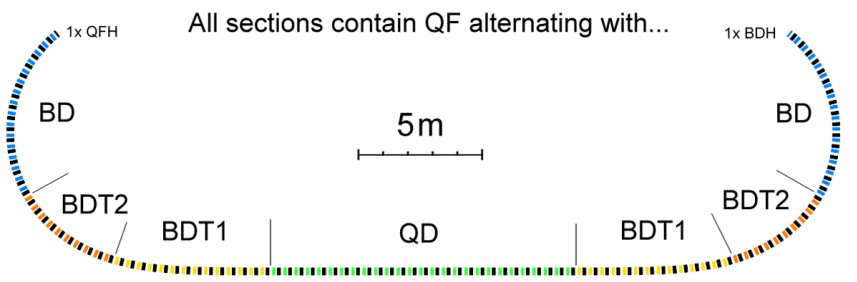

FIG. 2. The fixed-field return loop for the CBETA ERL.

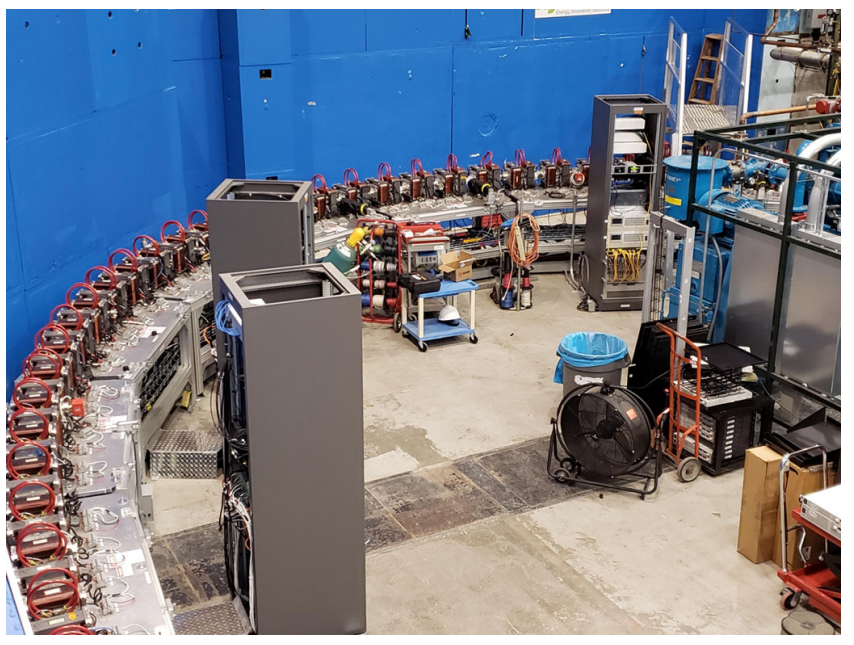

FIG. 3. Second arc of the installed CBETA return loop. 
TABLE II. Quality measures used for magnet acceptance.

\begin{tabular}{llc}
\hline \hline Quality measure & Limit & Units \\
\hline Maximum field error on midplane & $\leq 1.5$ & Gauss \\
Multipole FOM & $\leq 10$ & units \\
CBETA-scaled multipole FOM & $\leq 0.375$ & \\
Quadrupole strength error & $\leq 0.05$ & $\%$ \\
\hline \hline
\end{tabular}

wedge thicknesses to use are obtained via numerical optimization. Any modern optimization package that allows multiple input parameters and a multiple output variables to match to a vector of target values ought to work.

1. Optimizer inputs. For each wedge in the design there are a pair of parameters $(x, y)$, so there would be 32 parameters in total for the 16 wedge designs shown here. These are converted from Cartesian to polar coordinates $(r, \theta)$. The wedge thickness is set equal to $r$ and the magnetization angle is $\theta$, while the magnetization magnitude is the nominal value for the material chosen. The parameters may be reduced by a factor of two if horizontal midplane symmetry is enforced.

2. Optimizer outputs. The table of multipole amplitudes at the nominal good field radius ( $25 \mathrm{~mm}$ here) is the output used for optimization. Both the normal and skew poles from dipole up to 40-pole are considered here, making 40 output parameters. The goal vector is mostly zeroes apart

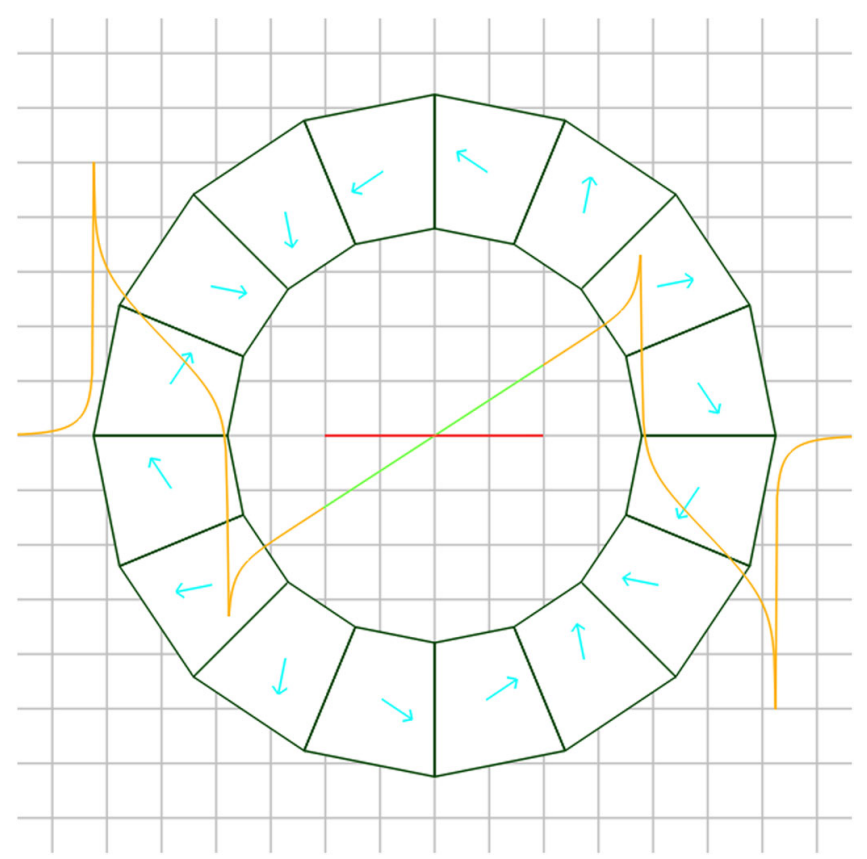

FIG. 4. Cross section of the quadrupole 'QF' magnet. Blue arrows show magnetization direction of the PM blocks. The orange line graphs the midplane field $B_{y}(x, 0)$, with green highlighting the good field region and red showing the beam position range in the FFA. The grid has $1 \mathrm{~cm}$ spacing.

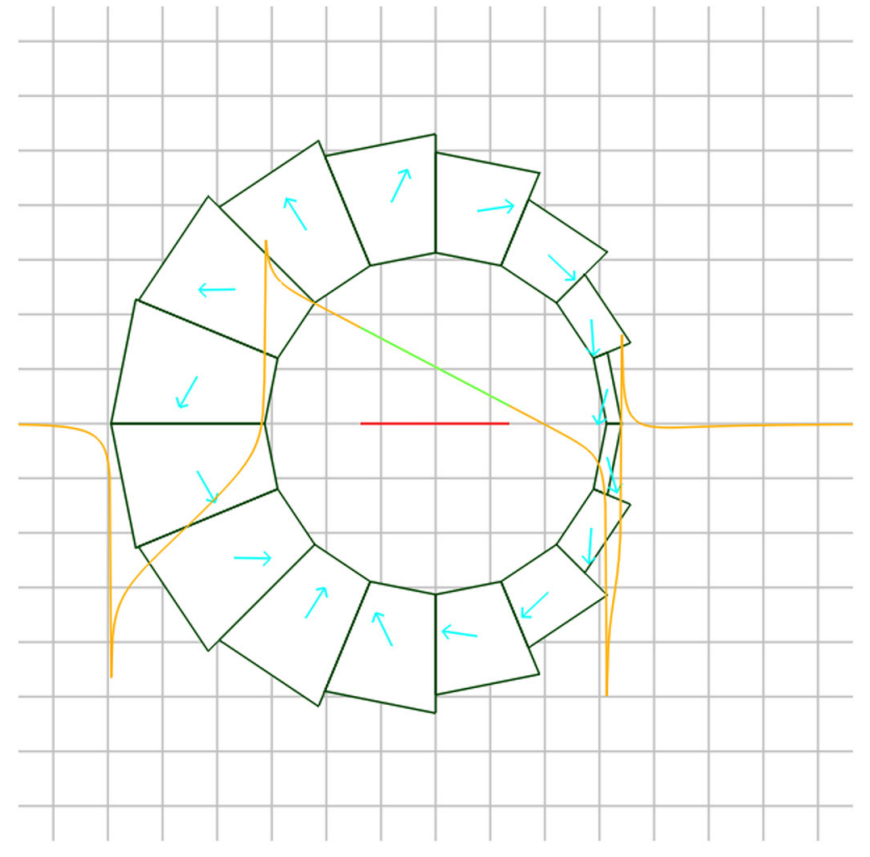

FIG. 5. Cross section of the BD magnet.

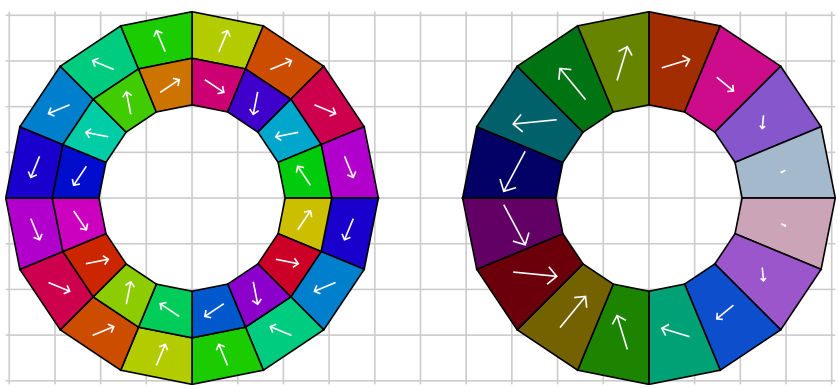

FIG. 6. Left: Halbach quadrupole nested inside a dipole. Right: vector sum of magnetization vectors in a single layer.

from the normal dipole and quadrupole strengths required from the magnet.

During optimization, the fields are evaluated using a slightly approximate but very fast 2D current sheet model, described in the next section. Typically these designs (without any errors) converged to within $10^{-5}$ relative field error in the optimizer.

In summary, this magnet design produces a straightforward generalization of the original circular magnets of Halbach [4], allowing variable radial wedge thicknesses and magnetization directions. Its applicability is not limited to just dipole + quadrupole but any combination of normal and skew multipoles provided they are not too strong for the material to provide. This includes the fields for scaling FFA machines (two examples were presented in [8]). Generalizing Halbach magnets is not a new idea: in fact, the general optimal case has been solved in recent work by Insinga [9]. However, that solution is so general that the PM 
pieces can have curved edges; in the interests of manufacturability, the magnets for CBETA were restricted to having straight edges and regular angles.

\section{B. Current sheet model}

The field calculation for these designs starts with Maxwell's magnetostatic equations in a material:

$$
\nabla \cdot \mathbf{B}=0 \quad \nabla \times \mathbf{B}=\mu_{0}(\mathbf{J}+\nabla \times \mathbf{M}) .
$$

It is approximated that the magnetization $\mathbf{M}$ does not vary with applied field, i.e. that the PM material has $\mu_{r}=1$. It is also assumed that each PM block has a constant $\mathbf{M}$ vector. This means that on a boundary with outward unit normal $\hat{\mathbf{n}}$, the magnetization is equivalent to a surface current

$$
\mathbf{j}_{s}=-\hat{\mathbf{n}} \times \mathbf{M} .
$$

Each edge of a polygonal PM block therefore produces a sheet current, which in the 2D approximation extends infinitely in $z$. The field of this sheet can be derived starting from the well-known field of an infinite wire with current $I$ in the $z$ direction:

$$
\mathbf{B}_{\text {wire }}(x, y ; I)=\frac{\mu_{0} I}{2 \pi r} \mathbf{e}_{\theta}=\frac{\mu_{0} I}{2 \pi}\left[\begin{array}{c}
-y /\left(x^{2}+y^{2}\right) \\
x /\left(x^{2}+y^{2}\right)
\end{array}\right],
$$

with $r, \theta$ being cylindrical polar coordinates. If the sheet has surface current density $j_{s}$ and extends from $(x, y)=$ $(0,0)$ to $(a, 0)$, then its field is the integral

$$
\begin{aligned}
\mathbf{B}(x, y) & =\int_{0}^{a} \mathbf{B}_{\text {wire }}\left(x-b, y ; j_{s}\right) \mathrm{d} b \\
& =\frac{\mu_{0} j_{s}}{2 \pi}\left[\begin{array}{c}
-\arctan (x / y)+\arctan ((x-a) / y) \\
\frac{1}{2}\left(\log \left(x^{2}+y^{2}\right)-\log \left((x-a)^{2}+y^{2}\right)\right)
\end{array}\right] .
\end{aligned}
$$

This field can be rotated and translated to place the sheet on any line in $(x, y)$ space. The sum can then be taken of a sheet current for each edge of the PM block to get the field of the entire polygon.

The strength is set via $\mu_{0}|\mathbf{M}|=B_{r 1}$, where $B_{r 1}$ is an approximation to the true remnant field $B_{r}$ of the material. A value $B_{r 1}<B_{r}$ is used to compensate for the fact that $\mu_{r} \simeq 1.02-1.05$ in reality, which reduces $|\mathbf{M}|$. This tuning was done by benchmarking against an OPERA-3D simulation using the manufacturer's B-H curve. The magnet was made using AllStar Magnetics grade N35EH, which is quoted to have $B_{r}=1.17-1.22 \mathrm{~T}$. Simulations used $B_{r 1}=1.15791 \mathrm{~T}$, which is derived from the low-end of the strength range, reduced by the expected temperature coefficient and difference between operating and measurement temperature.

\section{MULTIPOLE CANCELLATION WITH IRON RODS}

PM blocks as-manufactured have a random distribution of $\mathbf{M}$ vector errors with $|\delta \mathbf{M}| /|\mathbf{M}|$ typically of the order $10^{-2}$. One known method to correct this is block sorting, in which $\mathbf{M}$ is measured for all blocks and they are paired off to cancel errors when integrated through the longitudinal direction. However, construction errors in block position and angle will also produce significant field errors that would not be corrected by block sorting. Instead, a tuning method was devised for CBETA that corrects both these error sources in a single process after the bare magnet is manufactured [10]. Based on shimming but with the iron "floating" because there is no iron pole, this technique provided sufficient range to cancel both sources of error at once [11]. This generous tuning range also allowed cost savings during manufacture, through looser tolerances and only having to measure the magnetization of a representative sample of each block type.

A similar technique of floating iron shims to cancel multipole errors was used by Gupta $[12,13]$ in the RHIC interaction region superconducting quadrupoles. Other techniques exist to cancel errors in permanent magnet assemblies, such as mechanical tuning [14,15], although for CBETA this was felt to be more complex to manufacture than the packs of iron rods.

\section{A. Field perturbation from an iron rod}

When an iron rod, infinite in $z$ with a small circular cross section of radius $r_{\text {rod }}$, is inserted into a locally uniform magnetic field $\mathbf{B}_{0}$, it becomes magnetized, producing its own field that adds to the external one. Assuming perfect iron with $\mu_{r}=\infty$, the field lines must change to be perpendicular to the surface of the iron. Without loss of generality, assume that the external field is in the $x$ direction $\mathbf{B}_{0}=\left(B_{0 x}, 0\right)$ and write external dipole solutions to the $2 \mathrm{D}$ free space Maxwell's equations as

$\mathbf{B}_{\text {dip }}(x, y ; k)=\frac{k}{r^{2}}\left[\begin{array}{c}\cos (2 \theta) \\ \sin (2 \theta)\end{array}\right]=\frac{k}{r^{2}}\left[\begin{array}{c}2 \cos ^{2} \theta-1 \\ 2 \cos \theta \sin \theta\end{array}\right]$,

using the double angle formulae. By choosing the dipole source strength $k=r_{\text {rod }}^{2}\left|\mathbf{B}_{0}\right|$, the total field at the rod surface becomes

$$
\begin{aligned}
\mathbf{B}_{\text {surf }}(\theta) & =\mathbf{B}_{0}+\mathbf{B}_{\text {dip }}\left(r_{\text {rod }} \cos \theta, r_{\text {rod }} \sin \theta ; r_{\text {rod }}^{2}\left|\mathbf{B}_{0}\right|\right) \\
& =\left[\begin{array}{c}
B_{0 x} \\
0
\end{array}\right]+\left|\mathbf{B}_{0}\right|\left[\begin{array}{c}
2 \cos ^{2} \theta-1 \\
2 \cos \theta \sin \theta
\end{array}\right] \\
& =B_{0 x}(2 \cos \theta)\left[\begin{array}{c}
\cos \theta \\
\sin \theta
\end{array}\right]=B_{0 x}(2 \cos \theta) \mathbf{e}_{r},
\end{aligned}
$$


which is entirely radial and thus perpendicular to the iron surface. This means the choice of source strength $k$ was the correct one and the field contribution from the rod in a horizontal field is

$\mathbf{B}_{\text {rod }, \mathrm{x}}(x, y)=\mathbf{B}_{\text {dip }}\left(x, y ; r_{\text {rod }}^{2}\left|\mathbf{B}_{0}\right|\right)=\frac{r_{\text {rod }}^{2} B_{0 x}}{r^{2}}\left[\begin{array}{c}\cos (2 \theta) \\ \sin (2 \theta)\end{array}\right]$.

Using slightly different double angle formulas gives this expression in Cartesian coordinates:

$$
\begin{aligned}
\mathbf{B}_{\mathrm{rod}, \mathrm{x}}(x, y) & =\frac{r_{\mathrm{rod}}^{2} B_{0 x}}{r^{2}}\left[\begin{array}{c}
\cos ^{2} \theta-\sin ^{2} \theta \\
2 \cos \theta \sin \theta
\end{array}\right] \\
& =\frac{r_{\mathrm{rod}}^{2} B_{0 x}}{r^{4}}\left[\begin{array}{c}
x^{2}-y^{2} \\
2 x y
\end{array}\right] \\
& =\frac{r_{\text {rod }}^{2}}{\left(x^{2}+y^{2}\right)^{2}} B_{0 x}\left[\begin{array}{c}
x^{2}-y^{2} \\
2 x y
\end{array}\right] .
\end{aligned}
$$

The entire problem can be rotated by $90^{\circ}$ to get an expression for the field contribution from a rod in a purely vertical field with $\left|\mathbf{B}_{0}\right|=B_{0 y}$ :

$$
\begin{aligned}
\mathbf{B}_{\text {rod, } y}(x, y) & =R_{90^{\circ}} \mathbf{B}_{\text {rod }, \mathrm{x}}(y,-x) \\
& =\frac{r_{\text {rod }}^{2}}{\left(x^{2}+y^{2}\right)^{2}} B_{0 y} R_{90^{\circ}}\left[\begin{array}{c}
y^{2}-x^{2} \\
-2 x y
\end{array}\right] \\
& =\frac{r_{\text {rod }}^{2}}{\left(x^{2}+y^{2}\right)^{2}} B_{0 y}\left[\begin{array}{c}
2 x y \\
y^{2}-x^{2}
\end{array}\right] .
\end{aligned}
$$

Finally, as both of these solutions produce total fields perpendicular to the iron surface, they may be added together to get the general field contribution from a rod in an external field $\mathbf{B}_{0}=\left(B_{0 x}, B_{0 y}\right)$ :

$\mathbf{B}_{\text {rod }}(x, y)=\frac{r_{\text {rod }}^{2}}{\left(x^{2}+y^{2}\right)^{2}}\left[\begin{array}{c}B_{0 x}\left(x^{2}-y^{2}\right)+B_{0 y} 2 x y \\ B_{0 x} 2 x y+B_{0 y}\left(y^{2}-x^{2}\right)\end{array}\right]$.

In this solution, the iron is magnetized with $\mathbf{M}=2 \mathbf{B}_{0}$ and has surface currents in the a pattern of a " $\cos \theta$ " dipole. This formula may now be added on to the simulated field of a permanent magnet to model the effect of inserting iron rods.

\section{B. Rod lengths calculation}

Assembled magnets such as those in Fig. 9 were measured on BNL's rotating coil and the observed errors added on to the 2D field model. Then 64 iron rods were added to the simulation just inside the magnet bore and their cross-sectional areas optimized in order to reduce the harmonic errors to zero again. This used the same multiparameter optimizer as was used to design the shape of the combined function magnets.

1. Optimizer inputs. The cross sectional areas of the 64 iron rods in the simulation, which vary in radii. The areas have an upper limit corresponding to the full cross sectional area of the real rods.

2. Optimizer outputs. The table of multipole amplitudes from the simulation at the good field radius, both normal and skew, from dipole up to 40-pole, making 40 output parameters in total. The field is calculated as the simulated field of the PM pieces and the iron rods in that field, plus the error field derived from the rotating coil measurement. The goal vector is the correct multipole table of the magnet as in the spec.

The error fields for CBETA's magnets were canceled almost exactly in this optimization when rods up to $80 \mathrm{mil}$ $(2.03 \mathrm{~mm})$ diameter were allowed. The rod areas in 2D represent fractional lengths of $80 \mathrm{mil}$ wire in 3D for ease of production, the real lengths are given by

$$
\frac{l_{\text {rod }}}{l_{\text {magnet }}}=\frac{A_{\text {rod,sim }}}{A_{\text {rod,real }}} \Rightarrow l_{\text {rod }}=l_{\text {magnet }} \frac{r_{\text {rod,sim }}^{2}}{r_{\text {rod,real }}^{2}} .
$$

\section{Proof of tuning completeness}

A useful property of this tuning process is that it is "complete" in the sense it can correct any 2D harmonic, normal or skew. This can be proved by defining the complex field $B=B_{y}+i B_{x}$ and coordinate $z=x+i y$, in which terms the rod field formula simplifies to

$$
B_{\mathrm{rod}}(z)=\frac{r_{\mathrm{rod}}^{2}\left(-\bar{B}_{0}\right)}{z^{2}},
$$

where $B_{0}=B_{0 y}+i B_{0 x}$. This can also be expressed in terms of the area of the $\operatorname{rod} A=\pi r_{\text {rod }}^{2}$ :

$$
B_{\text {rod }}(z ; A)=\frac{A}{\pi} \frac{-\bar{B}_{0}}{z^{2}} .
$$

Consider the continuous limit of a large number of rods inserted around a circle of radius $R$, with cross sectional area $a(\theta)$ per radian of angle. The correction field from this configuration would be

$$
\begin{aligned}
B_{\text {corr }}(z) & =\int_{0}^{2 \pi} B_{\text {rod }}\left(z-R e^{i \theta} ; a(\theta)\right) \mathrm{d} \theta \\
& =\int_{0}^{2 \pi} \frac{a(\theta)}{\pi} \frac{-\bar{B}_{0}(\theta)}{\left(z-R e^{i \theta}\right)^{2}} \mathrm{~d} \theta,
\end{aligned}
$$

If the main magnet field is a pure multipole $B_{0}=c_{n} z^{n}$, noting that this is evaluated at the rod position $R e^{i \theta}$, the correction field is 


$$
B_{\mathrm{corr}}(z)=\int_{0}^{2 \pi} \frac{a(\theta)}{\pi} \frac{-\bar{c}_{n} R^{n} e^{-i n \theta}}{\left(z-R e^{i \theta}\right)^{2}} \mathrm{~d} \theta
$$

This is linear in $a(\theta)$, so a single Fourier component $a(\theta)=$ $e^{i m \theta+i \phi}$ of the rod pattern can be considered. Using the rod position $w=R e^{i \theta}$ as a complex variable in the integral with the differential $\mathrm{d} w=i R e^{i \theta} \mathrm{d} \theta$, this can be reexpressed as a contour integral

$$
\begin{aligned}
B_{\mathrm{corr}, m}(z) & =\oint \frac{e^{i \phi} R^{-m} w^{m}}{\pi} \frac{-\bar{c}_{n} R^{2 n} w^{-n}}{(z-w)^{2}} \frac{\mathrm{d} w}{i w} \\
& =\frac{-\bar{c}_{n} e^{i \phi} R^{2 n-m}}{\pi i} \oint \frac{w^{m-n-1}}{(w-z)^{2}} \mathrm{~d} w \\
& =-2 \bar{c}_{n} e^{i \phi} R^{2 n-m}(m-n-1) z^{m-n-2},
\end{aligned}
$$

where Cauchy's integral formula (first derivative form) has been used to evaluate the integral assuming the evaluation point is inside the rods $(|z|<R)$ and $m-n-1 \geq 0$. If $m-n-1<0$, an additional pole is introduced that cancels the residue from the others and the integral evaluates to zero.

Practically, this formula means that if a multipole magnet of order $n$ (where $0=$ dipole, $1=$ quad...) has an error field of order $p$, it can be corrected by a rod harmonic with $m$ lobes, where $p=m-n-2$, i.e., $m=n+p+2$. This was done experimentally in early R\&D in 2015 when a PM quadrupole had its sextupole error corrected by introducing a five-lobed distribution of rods, with details given in Appendix A.

Any desired normal/skew phase of the correction field can be produced by adjusting $\phi$ to make $\bar{c}_{n} e^{i \phi}$ have the same phase as the error coefficient. Explicitly, if the error to be corrected is $B_{\mathrm{err}}=b_{p} z^{p}$, the required rod distribution harmonic is $a(\theta)=a_{m} e^{i m \theta}$ where

$$
\begin{aligned}
-b_{p} & =-2 a_{m} \bar{c}_{n} R^{2 n-m}(m-n-1) \\
\Rightarrow a_{m} & =\frac{b_{p}}{2 \bar{c}_{n} R^{2 n-m}(m-n-1)} .
\end{aligned}
$$

This rod distribution $a_{m} e^{i m \theta}$ is still a complex number and needs to be made real. Noting that any multiple of $e^{i(-m) \theta}$ may be added with zero change to the correction field since $(-m)-n-1<0$, the complex conjugate may be added, resulting in the real rod distribution

$$
\begin{aligned}
a(\theta) & =a_{m} e^{i m \theta}+\bar{a}_{m} e^{-i m \theta} \\
& =2\left(\operatorname{Re} a_{m}\right) \cos (m \theta)-2\left(\operatorname{Im} a_{m}\right) \sin (m \theta) .
\end{aligned}
$$

Finally, any constant may be added onto $a(\theta)$ since $0-n-1<0$, which can be used to make all the rod areas positive, for example
$a(\theta)=2\left|a_{m}\right|+2\left(\operatorname{Re} a_{m}\right) \cos (m \theta)-2\left(\operatorname{Im} a_{m}\right) \sin (m \theta)$.

These formulas can produce a distribution of rods analytically from the measured field error harmonics by adding up the contributions from each harmonic. However, peaks of this function may go beyond the available magnet length and the tuning capacity is increased if rods can be "traded off" against each other in a constrained multiparameter optimization, as described in the previous section.

\section{PRACTICAL DESIGN CONSIDERATIONS}

\section{A. Radiation protection}

In [16], Temnykh derives an empirical formula that relates the radiation damage rate of $\mathrm{NdFeB}$ magnetic material to the margin between its operating temperature and the temperature at which it would demagnetize. As the temperature margin becomes larger, the damage rate with radiation exposure becomes exponentially slower. The CBETA magnets operate at approximately room temperature but the demagnetising temperature can be increased by choosing a material grade with high intrinsic coercivity $\left(H_{c j}\right)$. The second-highest coercivity grade available $(\mathrm{EH})$ was chosen to avoid end-bin effects. At this grade, a reasonably-priced material for its strength grade was N35EH.

With this material, it was calculated in [17] that it would require $74 \mathrm{kGy}$ of radiation to reduced the magnetization by $1 \%$. A conservative exposure limit of $1 \mathrm{kGy}$ was chosen for the CBETA return loop to keep well within the tolerances in Table II (discussed in Sec. VI). The return loop was instrumented with $90 \mathrm{CsI}$ dosimeters reading out several times per second to monitor the dose given to the magnets. These were placed at points on the magnet surface nearest the beam pipe on the machine midplane and would close the beam source laser shutter if limits were exceeded. For high current $(>\mathrm{mA})$ operation, a shutdown system based on fast BLMs will be added to prevent large doses in short amounts of time.

\section{B. Temperature stabilization with water}

As the $\mathrm{NdFeB}$ material has a temperature coefficient of $-1.1 \times 10^{-3} / \mathrm{K}$, temperature-controlled water was circulated through channels in the magnets, both during field measurement and machine operation. A temperature of $29.4^{\circ} \mathrm{C}$ (nominally $85^{\circ} \mathrm{F}$ ) was maintained to within $\pm 0.2^{\circ} \mathrm{C}$. Results from a test are shown in Fig. 7, where the relative magnet strength varies by less than $10^{-4}$ once temperature control is established. 


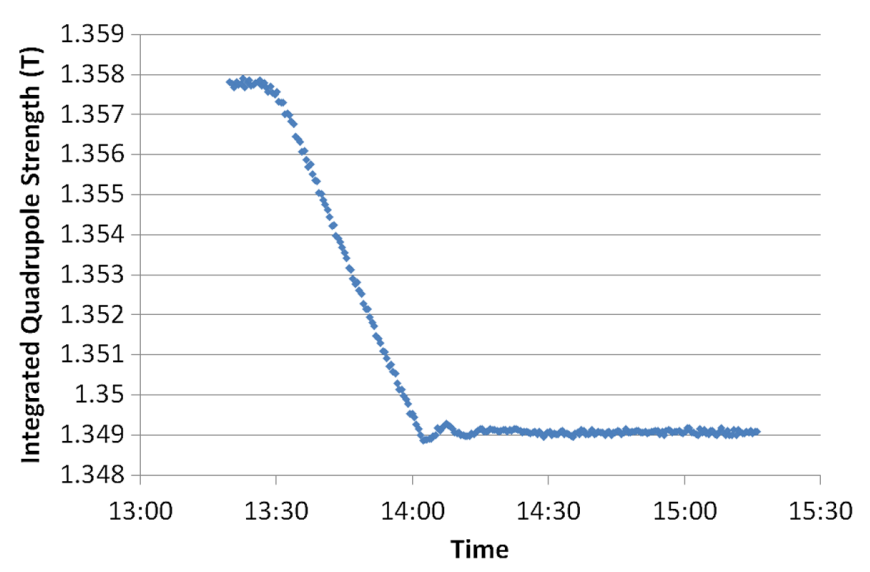

FIG. 7. Measured strength of a QD quadrupole as its temperature is stabilized from room temperature to $29.4{ }^{\circ} \mathrm{C}$.

\section{PRODUCTION}

Each full-length magnet contains 32 permanent magnet wedges: two layers of the designs shown in Fig. 1. The BDtypes require 16 distinct types of wedge when magnetization angles are also considered, whereas the Q-type magnets can be built from four types of wedge, some inserted backwards. In total there are 56 different wedge types and 7648 wedges (including spares), which were ordered from Allstar Magnetics [18] and produced in China. RMS magnetization accuracies of $1 \%$ strength and $1^{\circ}$ angle were achieved for most wedges, with larger angle tolerances allowed for the smallest two BD-type wedges, which are more difficult to manufacture and contribute less to the total field. Quality control was achieved with the testing process in Fig. 8.

Manufacture of the aluminium frames and gluing the magnet wedges into them was done by KYMA [19], who had previous experience with making undulator magnets and the strong forces between permanent magnet blocks. KYMA's metrology of the aluminium frames indicated corner positions were correct to within $\pm 0.1 \mathrm{~mm}$ before insertion of the PM wedges. These had to be positioned

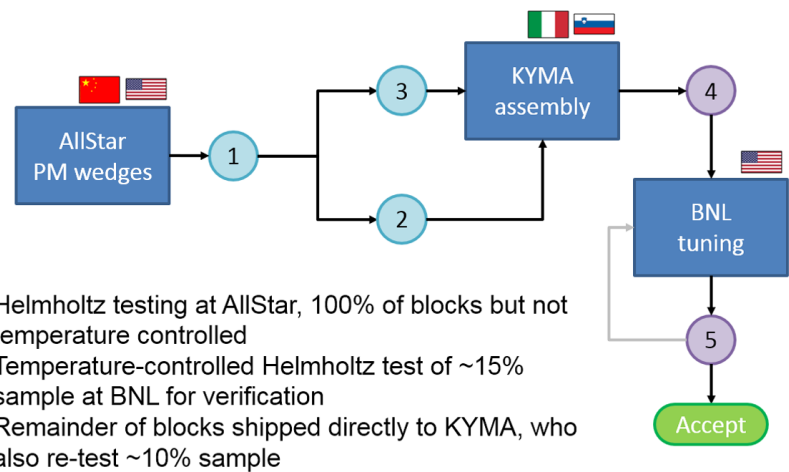
also re-test $\sim 10 \%$ sample

4. Rotating coil measurement of bare magnet at BNL

5. Rotating coil measurement of tuned magnet at BNL

FIG. 8. Production and testing flow. accurately enough to ensure good initial field quality and this was achieved. Field strength could be re-tuned during production by changing the thickness of brass shims inserted between the wedges, which was done successfully for the QD magnets. The frames were made in left-right halves with pins to accurately align them with each other when assembled onto the vacuum chamber. Threaded rods had to be used to overcome repulsive and attractive forces [20] during this assembly.

(a)

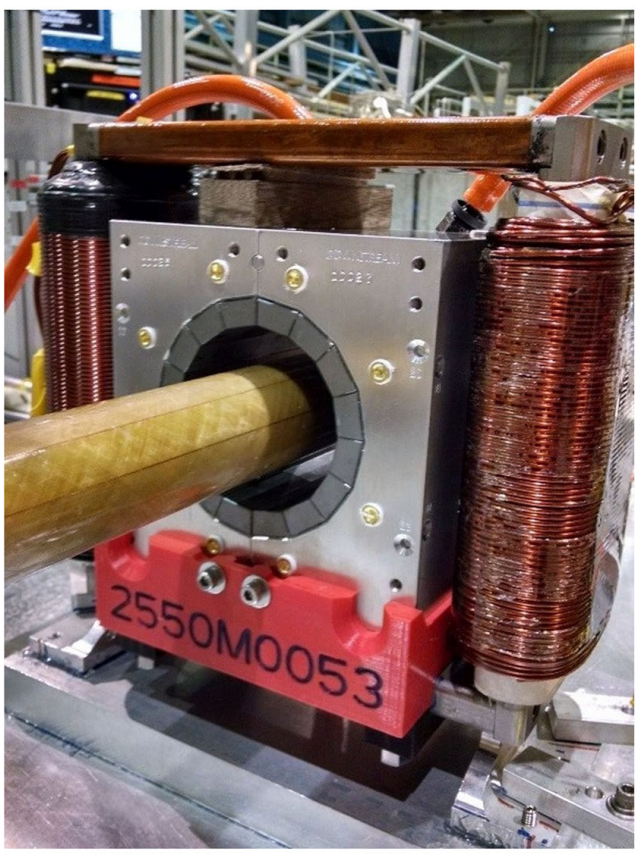

(b)

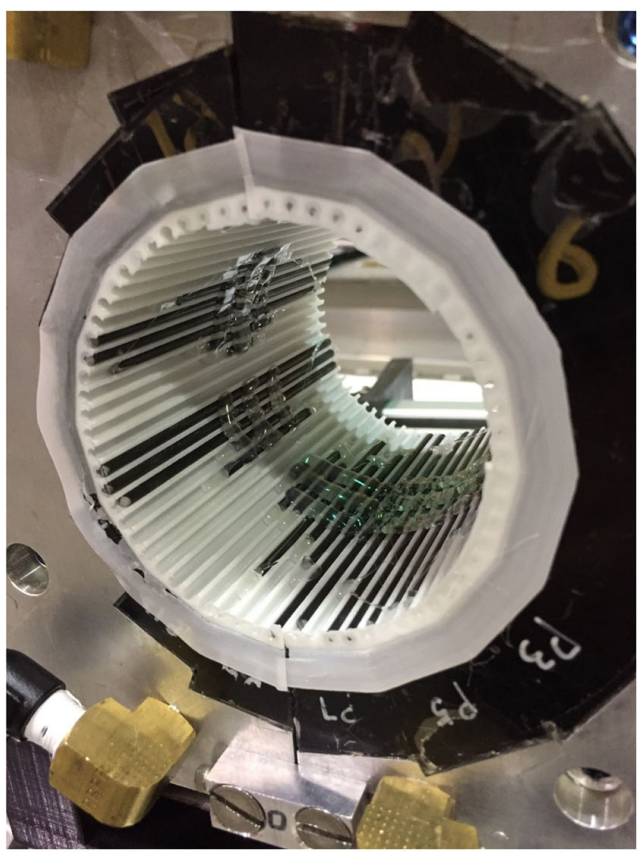

FIG. 9. (a) QD magnet being measured with a rotating coil at BNL magnet division. (b) BD magnet with tuning rod pack inserted. 


\section{A. Magnet tuning procedure}

Once the magnets reached BNL, they were measured on a rotating coil and then tuned using the method in Sec. III and [10]. 3D printed plastic tuning packs [21] containing the iron rods were inserted to cancel the multipole errors and the magnet was measured again to verify. Figure 9 shows two steps in this process. The iron rods were 10061008 carbon steel supplied in $1 \mathrm{ft}$ straight lengths.

Locations on the magnet and rotating coil were surveyed during each measurement to relate the field center to the fiducials used by the surveyors during installation. The procedures for combined measurement and survey are documented in [22].

Results were recorded on a network drive with systematic filenames defined by [23] so that an automated tool could process the data. This tool took the magnet and measurement run numbers as input and would output either that the magnet had passed the acceptance criteria in Table II, or a list of rod lengths to be used for tuning. The tool could be run by the several technicians manufacturing the magnets without physicist or programmer intervention. The tuning rods were measured by hand and cut to length by a pneumatic cutter before being hot glued into the tuning pack. Fully robotic cutting was attempted but the large $\sim 2 \mathrm{~mm}$ diameter of the iron made straightening it from a continuous spool difficult, so semimanual cutting plus a manual quality check of the lengths was found to be workable.

The schedule achieved for the production run of the CBETA permanent magnets is given in Appendix B.

\section{FIELD QUALITY RESULTS}

Several field quality metrics were calculated for each magnet from rotating coil data and compared to the acceptance thresholds given in Table II.

The maximum field error is measured on the $y=0$ midplane good field region, which is $\pm 25 \mathrm{~mm}$ in $x$. All field values are averages derived by dividing the integrated field measured by the rotating coil by the nominal magnet length.

The multipole figure of merit $(\mathrm{FOM})$ is defined as

$$
\sqrt{\sum_{n \geq \operatorname{sext}} b_{n}^{2}+a_{n}^{2}}
$$

where $b_{n}$ and $a_{n}$ are the normal and skew harmonics, respectively, measured in "units" defined as $10^{-4}$ of the main quadrupole field at $R=25 \mathrm{~mm}$.

The CBETA-scaled multipole FOM is defined as

$$
\sqrt{\sum_{n \geq \operatorname{sext}}\left(\frac{b_{n}}{b_{\lim , n}}\right)^{2}+\left(\frac{a_{n}}{a_{\lim , n}}\right)^{2}},
$$

where the limits for each individual harmonic are derived from tracking studies with errors [24]. It was found the
TABLE III. Multipole harmonics during correction of QD Magnet 2412.

\begin{tabular}{lcccc}
\hline \hline$R=25 \mathrm{~mm}$ & \multicolumn{2}{c}{ Before } & \multicolumn{2}{c}{ After } \\
\hline Pole & Normal & Skew & Normal & Skew \\
\hline Quad & 10000 & $\ldots$ & 10000 & $\ldots$ \\
Sext & -2.46 & -7.64 & 1.23 & -0.16 \\
Oct & 10.09 & -3.52 & 0.02 & 1.74 \\
Deca & 5.30 & 3.48 & -1.76 & 0.39 \\
Dodeca & -36.67 & -0.09 & -0.47 & -2.90 \\
14-pole & -1.76 & 0.19 & 0.06 & 0.10 \\
16-pole & 0.80 & -0.52 & 0.20 & -0.08 \\
18-pole & -0.32 & 0.25 & 0.04 & -0.09 \\
20-pole & -0.69 & 0.92 & -0.37 & 0.00 \\
22-pole & -0.23 & -0.04 & -0.06 & -0.02 \\
24-pole & -0.01 & -0.12 & 0.02 & -0.07 \\
26-pole & 0.00 & 0.10 & 0.05 & 0.01 \\
28-pole & 0.05 & 0.04 & 0.02 & 0.03 \\
30-pole & 0.02 & -0.01 & -0.01 & -0.01 \\
32-pole & -0.01 & 0.00 & -0.01 & -0.02 \\
34-pole & 0.00 & -0.01 & 0.02 & -0.01 \\
36-pole & 0.25 & -0.01 & 0.10 & 0.05 \\
38-pole & 0.01 & 0.00 & 0.00 & 0.00 \\
40-pole & 0.01 & 0.01 & 0.00 & 0.00 \\
Midplane field error & 11.80 Gauss & 0.42 Gauss \\
Multipole FOM & 39.62 units & 4.08 units \\
CBETA FOM & \multicolumn{2}{c}{1.796} & 0.095 \\
Quad strength error & $-0.157 \%$ & $0.005 \%$ \\
\hline \hline
\end{tabular}

FOM had to be less than 0.75 to preserve beam quality under the assumption that the magnets were the only source of error, so half this value was used as the production limit.

An example of the multipole errors in a typical magnet before and after tuning is given in Table III, along with the four figures of merit defined above.

Figure 10 shows how the multipole FOM of magnets decreased during the tuning process to be uniformly better

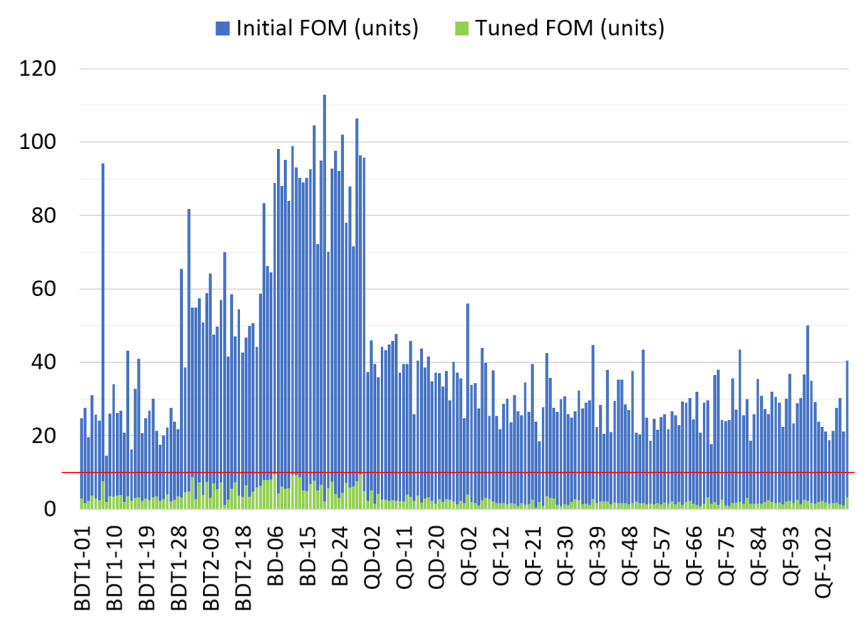

FIG. 10. Multipole FOM of magnets before and after tuning. 
TABLE IV. Multipole figure of merit statistics.

\begin{tabular}{lcc}
\hline \hline Multipole FOM (units) & Initial & Tuned \\
\hline Average & 41.09 & 3.09 \\
RMS & 46.92 & 3.70 \\
Maximum & 112.87 & 9.63 \\
Minimum & 14.64 & 0.52 \\
Median & 32.76 & 2.33 \\
\hline \hline
\end{tabular}

than the limit. Good results were also obtained for the quadrupole strength error (see [25] for more detail).

Table IV gives summary statistics for the multipole FOM of all CBETA magnets.

Figure 11 shows the CBETA FOM for all magnets, which also decreased to be uniformly below the limit of 0.375. As a bar chart, it looks similar to Fig. 10 but the scatter plot highlights some of the differences between magnet types. Quadrupoles (QF, QD) tend to have smaller errors because the reduced number of distinct PM wedge types and their symmetry cancels some of the systematic

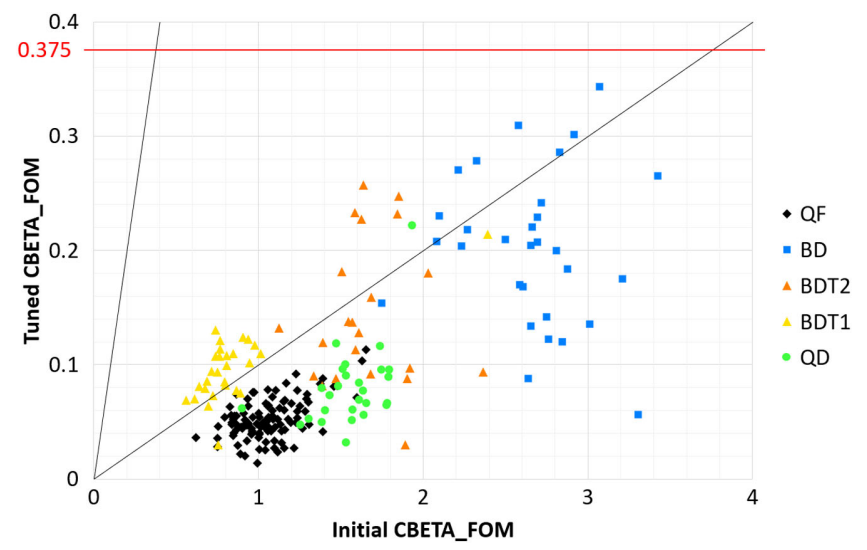

FIG. 11. CBETA FOM of magnet types before and after tuning. Lines for $1 \times$ and $10 \times$ improvement are shown.

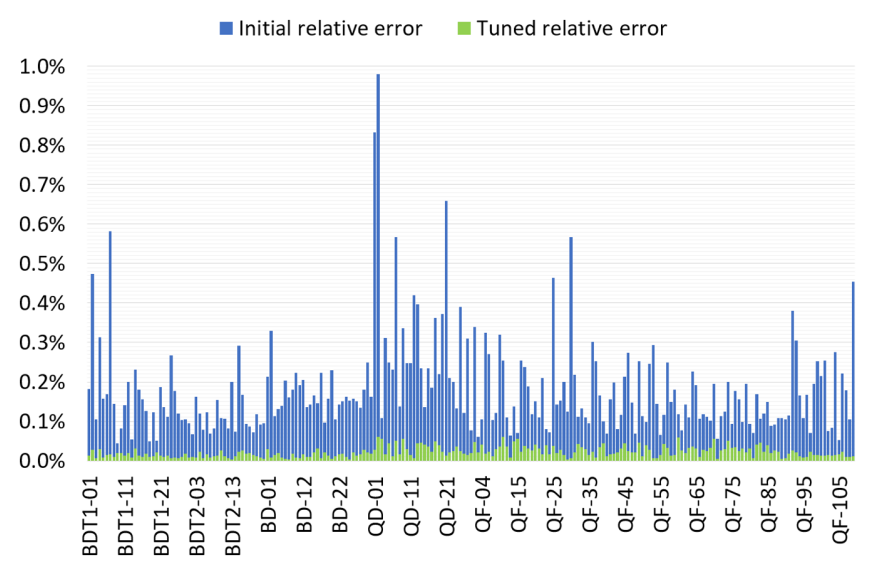

FIG. 12. Relative field errors before and after tuning.
TABLE V. CBETA scaled figure of merit statistics.

\begin{tabular}{lcc}
\hline \hline CBETA FOM & Initial & Tuned \\
\hline Average & 1.380 & 0.092 \\
RMS & 1.515 & 0.113 \\
Maximum & 3.422 & 0.343 \\
Minimum & 0.562 & 0.014 \\
Median & 1.157 & 0.070 \\
\hline \hline
\end{tabular}

TABLE VI. Relative field error statistics.

\begin{tabular}{lcc}
\hline \hline Relative field error & Initial & Tuned \\
\hline Average & $1.82 \times 10^{-3}$ & $2.19 \times 10^{-4}$ \\
RMS & $2.20 \times 10^{-3}$ & $2.56 \times 10^{-4}$ \\
Maximum & $9.81 \times 10^{-3}$ & $6.15 \times 10^{-4}$ \\
Minimum & $4.41 \times 10^{-4}$ & $3.05 \times 10^{-5}$ \\
Median & $1.50 \times 10^{-3}$ & $1.90 \times 10^{-4}$ \\
\hline \hline
\end{tabular}

magnetization angle errors. Larger aperture magnets (BDT1, BDT2, QF) also show lower errors at $R=$ $25 \mathrm{~mm}$ than comparable magnets with smaller apertures, which is to be expected since higher multipoles decay faster than the main field as the radius is reduced. Summary statistics for the CBETA FOM are given in Table V.

Figure 12 expresses the field quality as a relative error $\max \left|\mathbf{B}-\mathbf{B}_{\text {goal }}\right| / \max \left|\mathbf{B}_{\text {goal }}\right|$ taken over the midplane good field region.

Table VI gives summary statistics for the relative field error, with every tuned magnet better than $10^{-3}$ and the average relative field error over all tuned magnets being $2.2 \times 10^{-4}$.

\section{CONCLUSION}

The magnet production run was a success, producing all magnets with good field quality within the deadline. The cost per magnet for $\mathrm{NdFeB}$ material was $\$ 3303$ and the total cost fit within the planned CBETA budget. Note, however, that the cost of rare earth materials can vary substantially with fluctuations in global market supply.

Use of the magnets in the CBETA 2019-20 operating runs was also a success, as reported in [1], with good orbit through the return loop being achieved for all four energies simultaneously. Use of permanent magnets as the main bending field of an accelerator has been a rarity in the past (the Fermilab recycler being one of the main exceptions) and has never been done before in an ERL.

The method in this paper shows a cost-effective way of achieving accelerator-quality permanent magnets, using a single tuning method that corrects all sources of field error (magnetization, misplacement) at once. This method can be automated with software and handled by technicians during 
production. The tuning packs are simple and cheap to make.

Future applications of permanent magnets in accelerators include high-gradient quadrupoles for low-emittance light sources [26], which could be made using a variant of the methods in this paper but scaled down to smaller apertures. The rotating coil would likely be replaced by a rotating wire diagnostic and the plastic tuning packs replaced with thin tape upon which the proportionately-smaller iron rods would be adhered. A horizontal gap would be introduced to let synchrotron radiation exit without irradiating the magnet. High-gradient PM quadrupoles are also under consideration for focussing in laser-plasma accelerators [27] and PM-based hadron cancer therapy gantries [28].

\section{ACKNOWLEDGMENTS}

This work and the construction of CBETA was funded by the New York State Energy Research and Development Authority (NYSERDA). The author would like to thank Nick Tsoupas for designing the SmCo magnet pieces and Animesh Jain for doing the rotating coil measurements used in Appendix A.

\section{APPENDIX A: SEXTUPOLE CORRECTION TEST}

This section uses the mathematical notation defined in Sec. III C throughout.

An initial experiment was done during R\&D in 2015 to test the correction of just a single multipole. The permanent magnet quadrupole $(n=1)$ shown in Fig. 13 would have its sextupole error $(p=2)$ corrected. The results of Sec. III $\mathrm{C}$ imply that the correcting wire distribution has $m=$ $n+p+2=5$ lobes. A distribution of rods $a(\theta) \propto 1+$ $\cos (5 \theta+\phi)$ was introduced, which contains Fourier modes for $m=0,-5$ as well as the $m=5$ that does the tuning, but these other modes have $B_{\text {corr }, m}=0$.

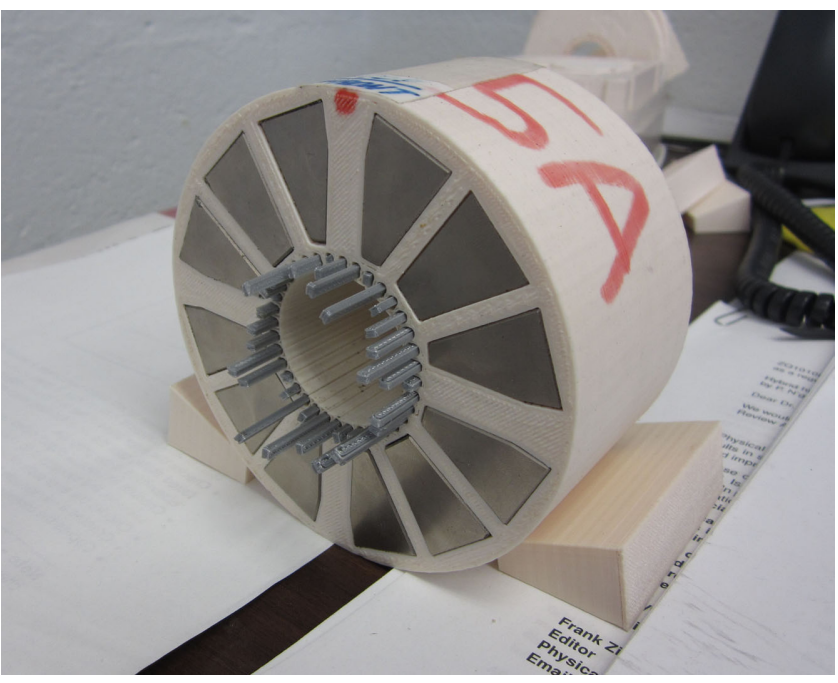

FIG. 13. R\&D permanent magnet quadrupole " $5 \mathrm{~A}$ ".
This magnet is made of SmCo material and the pieces are entirely enclosed in $3 \mathrm{D}$ printed $\mathrm{ABS}$ plastic. It has a quadrupole field of approximately $27.5 \mathrm{~T} / \mathrm{m}$ and a length of $l_{\text {magnet }}=60 \mathrm{~mm}$. The holes for the iron rods are also formed as part of the 3D print and there are $N_{\text {holes }}=36$ holes regularly spaced around the aperture, which keep the iron rods at $R=21 \mathrm{~mm}$ from the magnet centre. The grey plastic pieces seen protruding from these holes in Fig. 13 have wedges on the inner ends that are designed to clamp the iron rods against the outermost radius, for better consistency. This design was improved until CBETA has the rods completely filling a circular hole.

The corrections to this magnet's originally measured field were calculated using the current sheet model shown in Figure 14. The optimization method was similar to that explained in Sec. III B except only two variables were used: the amplitude and phase of the " $5 \theta$ " rod length distribution.

The magnet is quite small so rods of $14 \mathrm{mil}(0.356 \mathrm{~mm})$ diameter were used for correction, with a maximum length of $l_{\text {rod,max }}=119.2 \mathrm{~mm}$. This length is longer than the magnet so sometimes two wires were placed in the same hole to get the correct total length. This was later found to be a source of error because the wires in close proximity magnetically interact and change their total magnetization, so single wires per hole were exclusively used in CBETA. The amplitude of $a_{m}$ is one quarter of the maximum rod distribution, converted to area per radian, thus the value used in the experiment was

$$
\left|a_{m, \exp }\right|=\frac{1}{4} \frac{l_{\text {rod,max }}}{l_{\text {magnet }}} \pi r_{\text {rod }}^{2} \frac{N_{\text {holes }}}{2 \pi}=0.2826 \mathrm{~mm}^{2} / \mathrm{rad}
$$

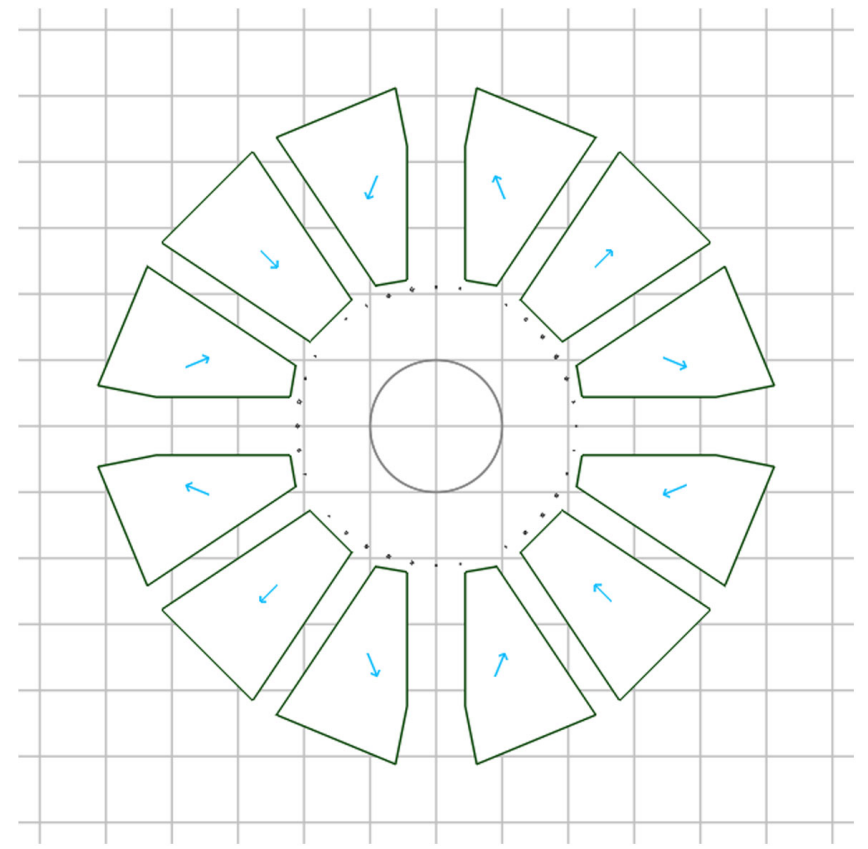

FIG. 14. Current sheet model of permanent magnet quadrupole " $5 \mathrm{~A}$ " with correction rod locations and $1 \mathrm{~cm}$ radius circle shown at center. 
TABLE VII. Multipole harmonics during correction of a sextupole error in a small R\&D PM quadrupole using a five-lobed rod distribution.

\begin{tabular}{|c|c|c|c|c|}
\hline \multirow{2}{*}{$\frac{R=10 \mathrm{~mm}}{\text { Pole }}$} & \multicolumn{2}{|c|}{ Before } & \multicolumn{2}{|c|}{ After } \\
\hline & Normal & Skew & Normal & Skew \\
\hline Quad & 10000 & $\ldots$ & 10000 & \\
\hline Sext & -19.46 & -6.42 & -0.58 & -0.63 \\
\hline Oct & 5.61 & -21.20 & 5.21 & -21.18 \\
\hline Deca & -0.99 & -4.02 & -0.84 & -4.23 \\
\hline Dodeca & -1.03 & 0.22 & -1.06 & 0.32 \\
\hline 14-pole & 1.25 & 0.07 & 1.04 & -0.16 \\
\hline 16-pole & -1.47 & -0.31 & -1.52 & -0.33 \\
\hline 18-pole & 0.12 & -0.05 & 0.13 & -0.06 \\
\hline 20-pole & 0.44 & 0.24 & 0.40 & 0.23 \\
\hline 22-pole & -0.03 & 0.00 & -0.01 & 0.01 \\
\hline 24-pole & 0.05 & -0.01 & 0.03 & -0.02 \\
\hline 26-pole & -0.01 & 0.00 & -0.01 & 0.01 \\
\hline 28-pole & -0.12 & 0.00 & -0.12 & 0.00 \\
\hline 30-pole & 0.00 & 0.00 & 0.00 & 0.00 \\
\hline \multicolumn{5}{|c|}{ Sextupole magnitude $\sqrt{b_{\text {sext }}^{2}+a_{\text {sext }}^{2}}$} \\
\hline
\end{tabular}

and its phase was

$$
\operatorname{Arg} a_{m, \exp }=\phi=-2.849 \mathrm{rad}
$$

The magnet was measured on a BNL rotating coil at reference radius $R_{\text {coil }}=10 \mathrm{~mm}$ and the results before and after correction are given in Table VII.

This choice of tuning rods cancelled all but $4.2 \%$ of the sextupole error in practice. The formula for $a_{m}$ in Sec. III C can now be used as a comparison. The initial magnet strength was measured to be

$$
c_{n}=27.502 \mathrm{~T} / \mathrm{m},
$$

so the complex error amplitude of the sextupole may be converted from units as

$$
\begin{aligned}
b_{p}= & (-19.46-6.42 i) 10^{-4} c_{n} R_{\text {coil }}^{n} / R_{\text {coil }}^{p} \\
= & (-5.352-1.766 i) \mathrm{T} / \mathrm{m}^{2} .
\end{aligned}
$$

The analytic prediction for the correction coefficient is

$$
\begin{gathered}
a_{m}=\frac{b_{p}}{2 \bar{c}_{n} R^{2 n-m}(m-n-1)} \\
=(-0.3004-0.0991 i) \mathrm{mm}^{2} / \mathrm{rad} .
\end{gathered}
$$

The phase

$$
\operatorname{Arg} a_{m}=-2.823 \mathrm{rad}
$$

agrees well with the experimental value but the amplitude

$$
\left|a_{m}\right|=0.3163 \mathrm{~mm}^{2} / \mathrm{rad}
$$

is $11.9 \%$ high. This means the real experiment used less rod area than predicted by the formula. This discrepancy is explained by the field at the some of the rod positions being up to $22 \%$ larger in the current sheet model compared to the pure quadrupole approximation. This is caused by proximity of the rods to the inner corners of the polygonal permanent magnet pieces, which produce a local field enhancements. The current sheet model is more accurate in these outer locations than trying to extrapolate high orders of the rotating coil data beyond their reference radius, which requires multiplying by a very high power of $r / R_{\text {coil }}$.

\section{APPENDIX B: SCHEDULE ACHIEVED}

This section describes the production schedule of the 214 permanent magnets for CBETA. Up to five prototypes of each magnet type were received and tested before approving final production, as shown in the early part of

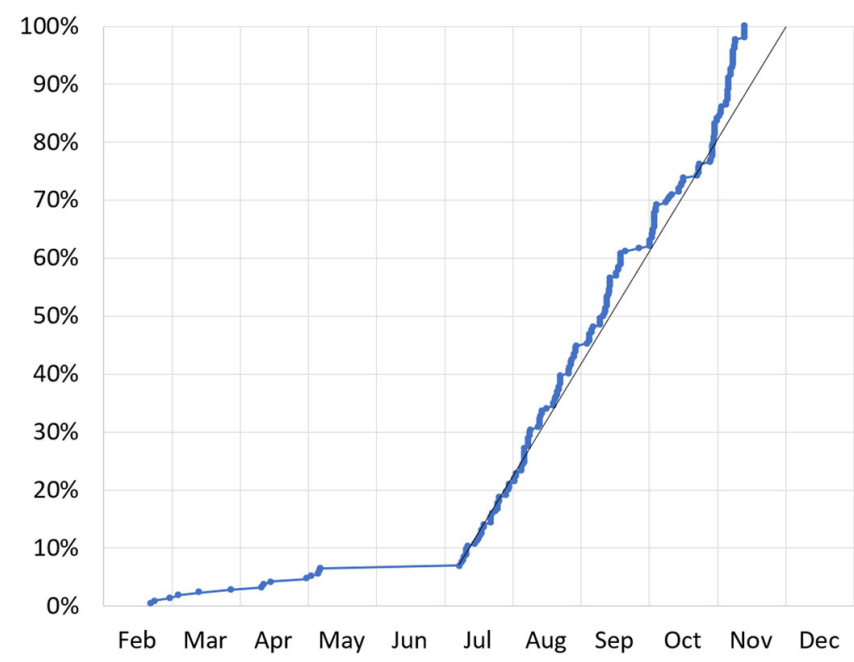

FIG. 15. Fraction of magnets completed over time.

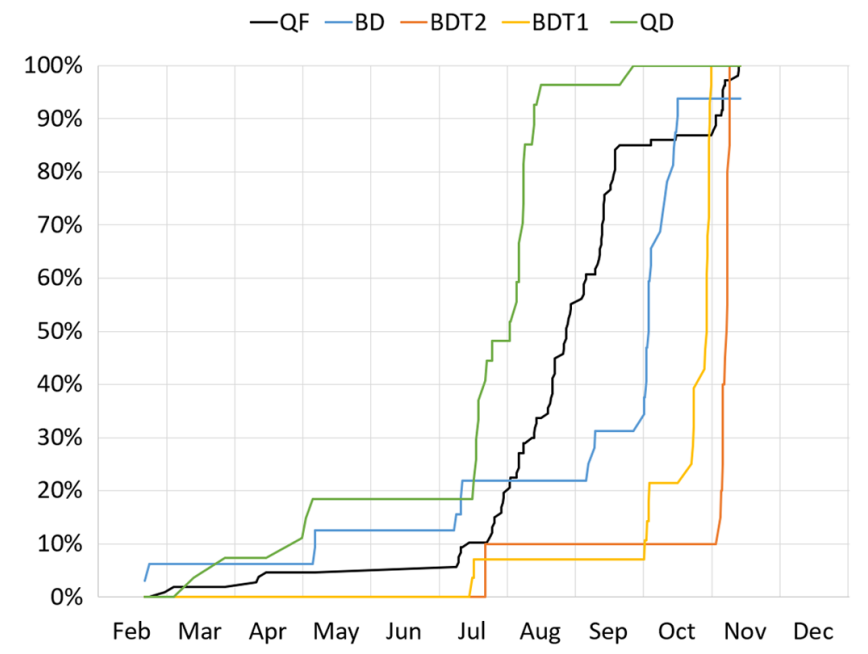

FIG. 16. Fraction of each magnet type completed over time. 
TABLE VIII. Main production run statistics.

\begin{tabular}{|c|c|}
\hline Magnets tuned & $\begin{array}{l}200 \\
\text { (excl. early samples) }\end{array}$ \\
\hline Rotating coil measurements & 545 \\
\hline Start date & July 9, 2018 \\
\hline End date & November 14, 2018 \\
\hline Total weeks & 18.2 \\
\hline $\begin{array}{l}\text { Magnets tuned per week } \\
\text { (avg.) }\end{array}$ & 10.96 \\
\hline $\begin{array}{l}\text { Rotating coil measurements } \\
\text { per week (avg.) }\end{array}$ & 29.88 \\
\hline $\begin{array}{l}\text { Rotating coil measurements } \\
\text { per magnet (avg.) }\end{array}$ & 2.73 \\
\hline
\end{tabular}

Fig. 15. There was then typically several weeks lead time before full production of the aluminium frames and magnets could occur, leading to the various magnet types being made in turns, as shown in Fig. 16.

The start of full production was defined by the first large batch of 27 magnets arriving at BNL and the statistics until completion are given in Table VIII. Two rotating coils were used in parallel for much of this time.

The diagonal line in Fig. 15 shows a linear interpolation from the full production start until the project milestone deadline of November 30th.

[1] A. Bartnik et al., CBETA: First Multipass Superconducting Linear Accelerator with Energy Recovery, Phys. Rev. Lett. 125, 044803 (2020).

[2] G. Hoffstaetter et al., CBETA design report, Cornell-BNL ERL test accelerator, arXiv:1706.04245.

[3] D. Trbojevic, E. D. Courant, and M. Blaskiewicz, Design of a nonscaling fixed field alternating gradient accelerator, Phys. Rev. Accel. Beams 8, 050101 (2005).

[4] K. Halbach, Design of permanent multipole magnets with oriented rare earth cobalt material, Nucl. Instrum. Methods 169, 1 (1980).

[5] P. Sarma, Ideal coil shape for combined function superconducting magnets, J. Phys. D 40, 3056 (2007).

[6] T. Nakamoto, N. Higashi, T. Ogitsu, A. Terashima, Y. Ajima, M. Anerella, R. Gupta, H. Hattori, T. Ichihara, Y. Iwamoto, N. Kimura, Y. Makida, T. Obana, K. ichi Tanaka, P. Wanderer, and A. Yamamoto, Design of superconducting combined function magnets for the $50 \mathrm{GeV}$ proton beam line for the J-PARC neutrino experiment, IEEE Trans. Appl. Supercond. 14, 616 (2004).

[7] H. Hahn and R. Fernow, Superconducting combined function magnets, IEEE Trans. Nucl. Sci. 30, 3402 (1983).

[8] S. Brooks, Generalized Halbach magnets for a non-scaling FFAG arc (2016), talk presented at FFAG2016 workshop, London; available from https://indico.cern.ch/event/ 543264/contributions/2295878/.

[9] A. Insinga, R. Bjrk, A. Smith, and C. Bahl, Globally optimal segmentation of permanent-magnet systems, Phys. Rev. Applied 5, 064014 (2016).
[10] S. Brooks, G. Mahler, J. Cintorino, and A. Jain, Production of low cost, high field quality Halbach magnets, in Proc. IPAC 2017 (2017), pp. 4118-4120, http://jacow.org/ ipac2017/papers/thpik007.pdf.

[11] S. Brooks, Error Studies of Halbach Magnets, CBETA note Report No. 010, 2017 available from https://www.classe. cornell.edu/CBETA_PM/index_notes.html.

[12] R. Gupta, M. Anerella, J. Cozzolino, B. Erickson, A. Greene, A. Jain, S. Kahn, E. Kelly, G. Morgan, P. Thompson, P. Wanderer, and E. Willen, Tuning shims for high field quality in superconducting magnets, IEEE Trans. Magnetics 32, 2069 (1996).

[13] R. Gupta, M. Anerella, J. Cozzolino, A. Ghosh, A. Jain, S. Kahn, E. Kelley, G. Morgan, J. Muratore, A. Prodell, W. Sampson, P. Thompson, P. Wanderer, and E. Willen, RHIC IR quadrupoles and field quality state of the art in superconducting accelerator magnets, in Proc. PAC 1999 (1999), pp. 185-187, http://accelconf.web.cern.ch/p99/ PAPERS/TUBR6.PDF.

[14] S. Herb, Construction and measurement of large permanent magnet quadrupoles, in Proc. PAC 1987 (1987), pp. 14341436, http://accelconf.web.cern.ch/p87/PDF/PAC1987_ 1434.PDF.

[15] S. Becker, M. Bussmann, S. Raith, M. Fuchs, R. Weingartner, P. Kunz, W. Lauth, U. Schramm, M. E. Ghazaly, F. Grner, H. Backe, and D. Habs, Characterization and tuning of ultrahigh gradient permanent magnet quadrupoles, Phys. Rev. Accel. Beams 12, 102801 (2009).

[16] A. Temnykh, Measurement of NdFeB permanent magnets demagnetization induced by high energy electron radiation, Nucl. Instrum. Methods Phys. Res., Sect. A 587, 13 (2008).

[17] S. Brooks, Radiation Limits for CBETA Halbach Magnets, CBETA note Report No. 036, 2018.

[18] Allstar Magnetics, https://allstarmagnetics.com/.

[19] Kyma S. r. 1., http://www.kyma-undulators.eu/.

[20] S. Brooks, Transverse Forces in the CBETA v6/v6.5 Magnets, CBETA note Report No. 017, 2017.

[21] S. Brooks, Description of CBETA Magnet Tuning Wire Holders, CBETA note Report No. 018, 2017.

[22] S. Brooks, Measurement Procedure for CBETA Halbach Magnets, CBETA note Report No. 019, 2017.

[23] S. Brooks, Data Directory for Halbach Magnets, CBETA note Repor No. 024, 2018.

[24] W. Lou, CBETA 4-pass Orbit Correction and Tolerance Study, CBETA note Report No. 037, 2018.

[25] S. Brooks, CBETA Halbach Magnet Production Run, CBETA note Report No. 035, 2018.

[26] G. Wang, T. Shaftan, V. Smaluk, Y. Hidaka, O. Chubar, T. Tanabe, J. Choi, S. Sharma, C. Spataro, and N. Mesentsev, Complex bend. II. a new optics solution, Phys. Rev. Accel. Beams 22, 110703 (2019).

[27] A. Ghaith, D. Oumbarek, C. Kitégi, M. Valléau, F. Marteau, and M.-E. Couprie, Permanent magnet-based quadrupoles for plasma acceleration sources, Instruments 3, 27 (2019).

[28] D. Trbojevic, S. Brooks, W. Lou, B. Parker, and N. Tsoupas, Permanent Halbach magnet proton and superconducting carbon cancer therapy gantries, in Proc. IPAC 2017 (2017), pp. 4679-4682, http://jacow.org/ ipac2017/papers/thpva094.pdf. 